\title{
GA and ACO-based Hybrid Approach for Continuous Optimization
}

\author{
Zhiqiang Chen* \\ Chongqing Engineering Laboratory for Detection Control \\ and Integrated System, School of Computer Science and \\ Information Engineering \\ Chongqing Technology and Business University \\ 19, Xuefu Avenue, Nan'an, Chongqing, 400067, China \\ *Corresponding author
}

\author{
Ronglong Wang \\ Country Graduate School of Engineering University of \\ Fukui \\ Fukui-shi, Japan 910-8507
}

\begin{abstract}
This paper presents an hybrid algorithm based on genetic algorithm and ant colony optimization for continuous optimization, which combines the global exploration ability of the former with the local exploiting ability of the later. The proposed algorithm is evaluated on several benchmark functions. The simulation results show that the proposed algorithm performs quite well and outperforms classical ant colony optimization and genetic algorithm for continuous optimization, which efficiently balances two contradictory aspects of its performance: exploration and exploitation.
\end{abstract}

Keywords-component; hybird; GA; ACO; continuous Optimization

\section{INTRODUCTION}

Many real-world application problems in engineering, science and technology can be formulated as continuous optimization problems (CnOPs) [1,2]. The continuous optimization problems always have local as well as global optima. Mostly, the user is interested in determining the global minima [3]. However, it is more difficult to determine the global minima rather than local minima for a lot of multimodal problems. As a result, most algorithms are very easy to be trapped in the local minima. Besides, for some non-separable benchmark problems, in view of the correlations information among the variables, the existing algorithms are difficult to converge to the global optimum, especially when the scale of the problem becomes larger [3,4].

Metaheuristics are a family of optimization techniques that have seen increasingly rapid development and have been applied to CnOPs over the past few years. Among them are genetic algorithm (GA) and ant colony optimization (ACO). GA has been used in many engineering applications since it was introduced as a robust and efficient search technique. The popularity of this method is based on simply solving multidimensional and multimodal optimization problems without requiring any additional information such as the gradient of an objective function. Although the origin of this method proposed binary number for encoding, over the past ten years, there were a surge of studies related to real-coded genetic algorithms (RCGA) for continue domain problem [3-6]. ACO is inspired by the ants' foraging behavior and it was first applied to solve discrete optimization problems [7-9]. The ant colony optimization was extended to the continuous domains by Socha [2], called $\mathrm{ACO}_{\mathrm{R}}$.

In this paper, we combine the conditionally breeding genetic algorithm model (CGA [4]) with $\mathrm{ACO}_{\mathrm{R}}[2]$ and develop a new hybrid algorithm for CnOPs. Several classical test problems available in the global optimization literature are used to test the performance of the proposed algorithm.

\section{METHODOLOGIES FOR CNOPS}

\section{A. Conditionally Breeding Genetic Algorithms model}

The original conditionally breeding genetic algorithms (CGA) is firstly defined in [10], in which crossover and mutation behaviors are performed by difference-degree between individuals instead of given probability. The CGA is a binary coded GA and thus it was applied to combinatorial optimization problems $[12,13]$. In the literature [4], we extend the CGA for continue optimization problems $\left(\mathrm{CGA}_{\mathrm{R}}\right)$. In the $\mathrm{CGA}_{\mathrm{R}}$, an important parameter controlling crossover and mutation is called setting difference-degree $D_{s}\left(0<D_{s}<1\right) . D_{s}$, which is decreased subsequent generation:

$$
D_{s}(t+1)=\mu D_{s}(t)
$$

where $t$ expresses $t^{\text {th }}$ generation, $\mu \in(0,1)$ is a constant variable called cooling ratio. The difference-degree between individuals is calculated as follows: Given two chromosomes $\mathbf{x}_{1}$ and $\mathbf{x}_{2}$, the difference-degree between $\mathbf{x}_{1}$ and $\mathbf{x}_{2}$ is defined as follows:

$$
d_{i}=\frac{\left\|\mathbf{e}_{1}-\mathbf{e}_{2}\right\|}{2}
$$

where $\mathbf{e}_{1}=\mathbf{x}_{1} /\left\|\mathbf{x}_{1}\right\|, \mathbf{e}_{2}=\mathbf{x}_{2} /\left\|\mathbf{x}_{2}\right\|$ and $\left\|\mathbf{e}_{1}-\mathbf{e}_{2}\right\|$ is the distance between $\mathbf{e}_{1}$ and $\mathbf{e}_{2}$. The criterion of crossover and mutation in CGA is by the difference-degree between or not by probability of conventional GA. 


\section{B. ACOR}

The first algorithm that can be classified as an ACO algorithm for continuous domains is $\mathrm{ACO}_{\mathrm{R}}[2]$. In $\mathrm{ACO}_{\mathrm{R}}$, the discrete probability distributions used in the solution construction by ACO algorithms for combinatorial optimization are substituted by probability density functions (PDFs). $\mathrm{ACO}_{\mathrm{R}}$ uses a solution archive [14] for the derivation of these PDFs over the search space. Additionally, $\mathrm{ACO}_{\mathrm{R}}$ uses sums of weighted Gaussian functions to generate multimodal PDFs. $\mathrm{ACO}_{\mathrm{R}}$ initializes the solution archive with $k$ solutions that are generated uniformly at random. Each solution is a $D$ dimensional vector with real-valued components $x_{t} \in\left[x_{\min }, x_{\max }\right]$, with $i=1,2, \ldots, D$. In this paper, we assume that the optimization problems are unconstrained except possibly for bound constraints of the $D$ real-valued variables $x_{i}$. The $k$ solutions of the archive are kept sorted according to their quality (from best to worst) and each solution $S_{j}$ has associated a weight $\omega_{j}$. This weight $\omega_{t}$ is calculated using a Gaussian function as [15]:

$$
\omega_{j}=\frac{1}{q k \sqrt{2 \pi}} e^{-\frac{(\operatorname{rank}(j)-1)^{2}}{2(q k)^{2}}}
$$

where $\operatorname{rank}(j)$ is the rank of solution $S_{j}$ in the sorted archive, and $q$ is a parameter of the algorithm. By computing $\operatorname{rank}(j)$, the best solution receives the highest weight. The weights are used to choose probabilistically a guiding solution around which a new candidate solution is generated. The probability of choosing solution $S_{j}$ as guiding solution is given by (2) [15]:

$$
p_{j}=\frac{w_{j}}{\sum_{r=1}^{k} w_{r}}
$$

So that the better the solution, the higher are the chances of choosing it. Once a guiding solution $S_{\text {gutde }}$ is chosen, the algorithm samples the neighborhood of the $i$-th real-valued component of the guiding solution $S_{\text {gutde }}^{l}$ using a Gaussian PDF with $S_{\text {gutde }}^{t}=\mu_{\text {gutde }}^{t}$, and $\sigma_{\text {gutde }}^{j}$ equal to

$$
\sigma_{\text {guide }}^{i}=\xi \sum_{r=1} \frac{\left|S_{r}^{i}-S_{\text {guide }}^{i}\right|}{k-1}
$$

which is the average distance between the value of the $i$-th component of $S_{\text {gutde }}^{l}$ and the values of the $i$-th components of the other solutions in the archive, multiplied by a parameter $\xi$ [15]. The process of choosing a guiding solution and generating a candidate solution is repeated a total of $N_{\mathrm{a}}$ times (corresponding to the number of "ants") per iteration. Before the next iteration, the algorithm updates the solution archive keeping only the best $k$ of the $k+N_{\text {a }}$ solutions that are available after the solution construction process.

\section{CGA and ACO-based Hybrid Approach}

Our past researches showed $\mathrm{CGA}_{\mathrm{R}}$ is a genetic algorithm with excellent ability of global search. However, it does not provide a good mechanism to tune the near-optimal in promising space for some non-separable function and multimodal problems. To improve the performance of $\mathrm{CGA}_{R}$ and balance between two contradictory aspects of their performance: exploration and exploitation, we utilize the exploiting mechanism of $\mathrm{ACO}_{\mathrm{R}}$ to develop a hybrid approach as Figure I. BLX- $\alpha$ crossover operator [16], and non-uniform mutation operator are used, which are as same as in the literature [4].

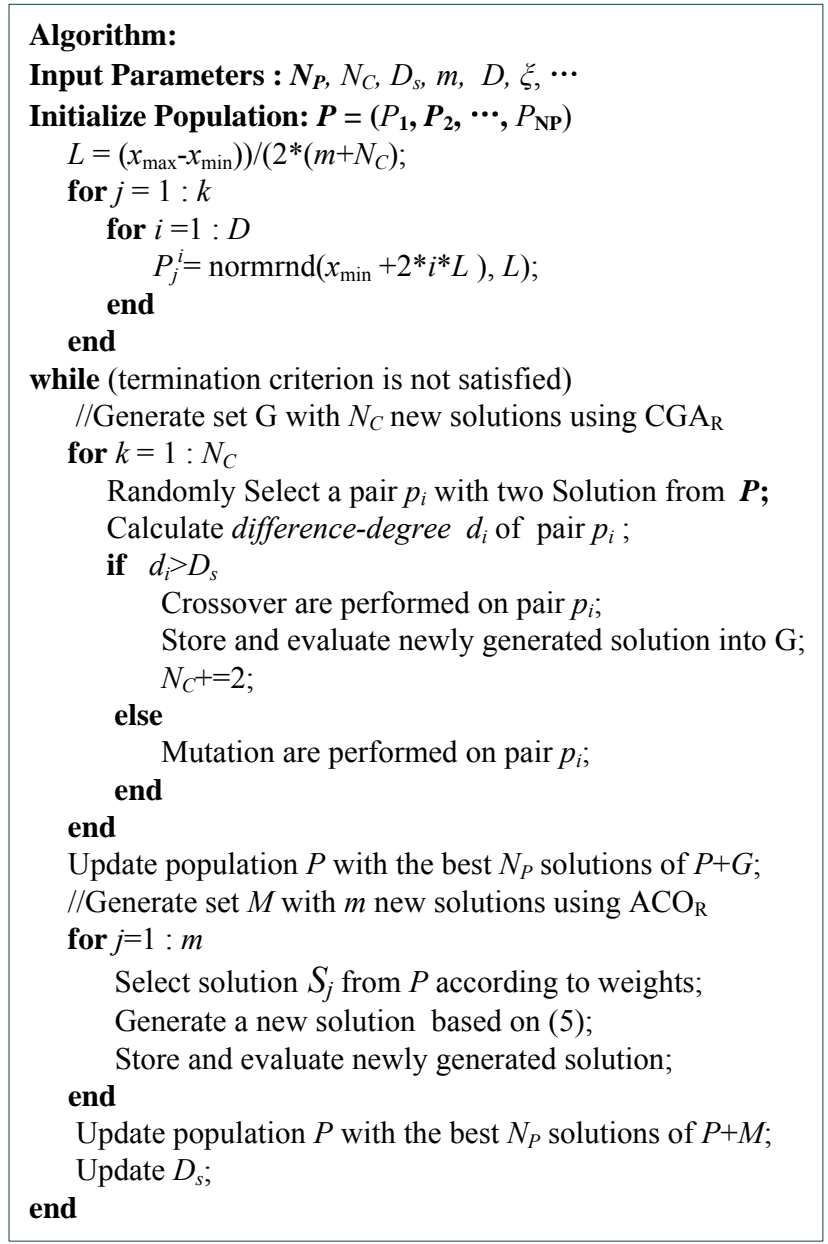

FIGURE I. HYBRID APPROACH BASED ON CGA AND ACOR

\section{EXPERIMENT AND DISCUSSION}

\section{A. Experimental setup}

In order to verify the effectiveness of the proposed algorithm, we use the following four test functions. Sphere function $f_{1}$ is the basic function to evaluate the algorithm [5]. For the non-separable function we choose Rosenbrock function $\left(f_{2}\right)[17]$. For the multimodal functions, the Schwefel function $\left(f_{3}\right)$ and the Rastrigin function $\left(f_{4}\right)$ are chosen. The dimensionality is set to 30 for all test functions. 
1. Sphere function

$$
\begin{aligned}
& \min _{x} f(x)=\sum_{i=1}^{n} x_{i}^{2}, \\
& -5.12 \leq x_{i} \leq 5.12, x^{*}=(0,0, \ldots, 0), f\left(x^{*}\right)=0 .
\end{aligned}
$$

2. Rosenbrock function

$$
\begin{aligned}
& \min _{x} f(x)=\sum_{i=2}^{n}\left(100\left(x_{1}-x_{i}^{2}\right)^{2}+\left(1-x_{i}\right)^{2}\right), \\
& -2.048 \leq x_{i} \leq 2.048, x^{*}=(0,0, \ldots, 0), f\left(x^{*}\right)=0 .
\end{aligned}
$$

3. Schwefel problem

$$
\begin{aligned}
& \min _{x} f(x)=418.9829 * n-\sum_{i=1}^{n} x_{i} \sin \left(\sqrt{\left|x_{i}\right|}\right), \\
& -500 \leq x_{i} \leq 500, \\
& x^{*}=(420.97,420.97, \ldots 420.97) \text { and } f\left(x^{*}\right)=0 .
\end{aligned}
$$

4. Rastrigin function

$$
\begin{aligned}
& \min _{x} f(x)=10 n+\sum_{i=1}^{n}\left(x_{i}^{2}-10 \cos \left(2 \pi x_{i}\right)\right), \\
& -5.12 \leq x_{i} \leq 5.12, x^{*}=(0,0, \ldots, 0), f\left(x^{*}\right)=0 .
\end{aligned}
$$

The parameters used in the proposed algorithm are as follows: population size $N_{P}$, the number of offspring generated by $\mathrm{CGA}_{\mathrm{R}}: N_{C}$, the number of offspring generated by exploit scheme: $m$, the parameter $\xi$ of standard deviation $\sigma_{j}^{j}$, the setting difference-degree $D_{s}$ and cooling ratio $\mu$. In this work, all parameters are set as follows: $N_{P}=180, N_{C}=30, \mathrm{~m}=30$, $\xi=0.76, D_{s}=0.1$ and $\mu=0.999$.

\section{B. Performance Evaluation}

To investigate the performance of the proposed algorithm, the convergence properties on four typical functions are analyzed, in comparison with the $\mathrm{CGA}_{\mathrm{R}}[3]$ and $\mathrm{ACO}_{\mathrm{R}}$ [2]. As shown Fig.2 5, the proposed hybrid approach can be fastest to find the global optimum than $\mathrm{CGA}_{R}$ and $\mathrm{ACO}_{\mathrm{R}}$.

For each test functions we performed 25 independent runs using each algorithm. The stopping criterions are as follows: $\left|f(s)-f\left(s^{*}\right)\right|<10^{-7}$ (s* is the global optimal solution), and the maximum number of function evaluations (MaxFEs) is set to $4 \mathrm{E}+6$. It means that if the error accuracy does not reach $10^{-7}$ within $4 \mathrm{E}+6 \mathrm{FEs}$, the simulation run is considered to an unsuccessful run. CGA $A_{R}+$ FPDD-LX [3] and the differential evolution (DE) [18] are employed to compare with the proposed algorithm. The $\mathrm{CGA}_{R}+\mathrm{FPDD}-\mathrm{LX}$ is another $\mathrm{CGA}_{\mathrm{R}}$ with local search mechanism. The DE is the state-of-the-art algorithm that is useful for the real world application, and we select the classical DE approach called DE/rand/1 to compare with the proposed algorithm. The mean numbers of the FEs of 25 independent runs for the above algorithm are recorded in Table 1. From the result in Table 1, we can see that the number of the FEs of the proposed algorithm is far fewer than $\mathrm{CGA}_{\mathrm{R}}$, $\mathrm{ACO}_{\mathrm{R}}, \mathrm{DE} / \mathrm{rand} / 1$ and $\mathrm{CGA}_{\mathrm{R}}+\mathrm{FPDD}-\mathrm{LX}$.

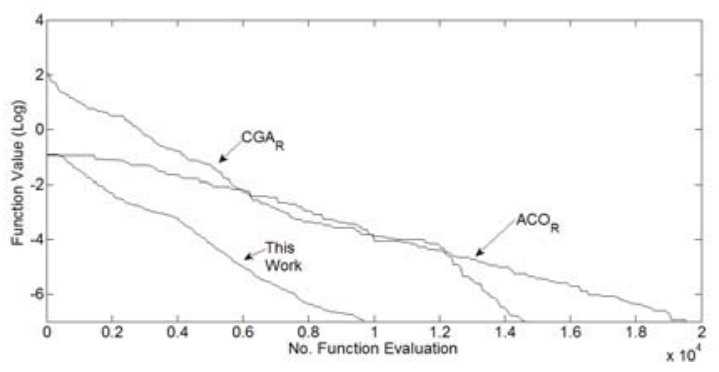

FIGURE II. THE CONVERGENCE PROCESS ON THE SPHERE FUNCTION

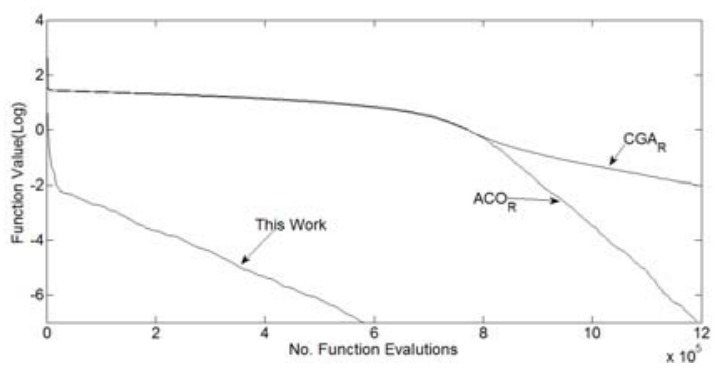

FIGURE III. THE CONVERGENCE PROCESS ON THE ROSENBROCK FUNCTION

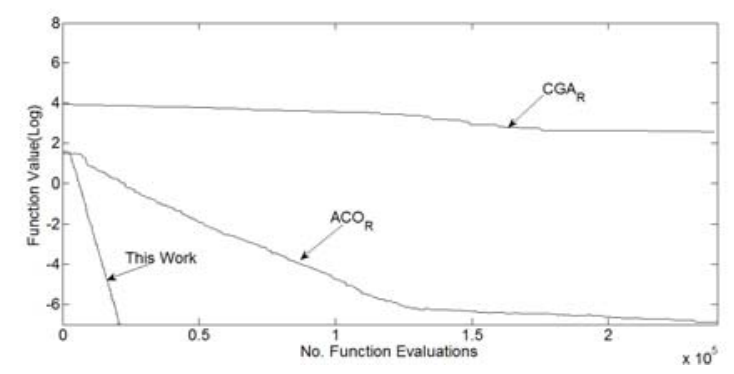

FIGURE IV. THE CONVERGENCE PROCESS ON THE SCHWEFEL FUNCTION

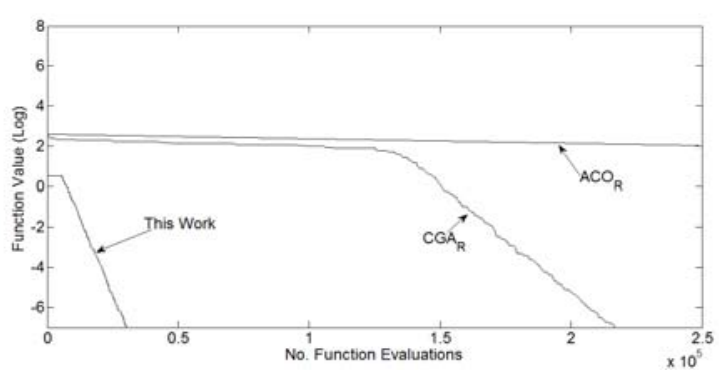

FIGURE V. THE CONVERGENCE PROCESS ON THE RASTRIGIN FUNCTION 
TABLE I. COMPARING WITH OTHER ALGORITHMS, D = 30

\begin{tabular}{|c|c|c|c|c|c|}
\hline Func & This work & $\mathbf{C G A}_{\mathbf{R}}$ & $\mathbf{A C O}_{\mathbf{R}}$ & $\mathbf{D E} / \mathbf{r a n d} / \mathbf{1}$ & $\begin{array}{c}\mathbf{C G A}_{\mathbf{R}}{ }^{+} \\
\mathbf{F P D D}_{\mathbf{L}} \mathbf{L}\end{array}$ \\
\hline$f_{1}$ & $1.31 \mathrm{e}+4$ & $1.36 \mathrm{e}+4$ & $1.84 \mathrm{e}+4$ & $4.39 \mathrm{E}+4$ & $\mathbf{1 . 2 6 E + 4}$ \\
\hline$f_{2}$ & $4.48 \mathrm{e}+5$ & -- & $1.20 \mathrm{e}+6$ & - & $3.40 \mathrm{E}+\mathbf{5}$ \\
\hline$f_{3}$ & $\mathbf{1 . 9 3 e}+\mathbf{4}$ & -- & $2.54 \mathrm{E}+5$ & $5.0 \mathrm{E}+5$ & $7.43 \mathrm{E}+5$ \\
\hline$f_{4}$ & $\mathbf{2 . 3 8 e}+\mathbf{4}$ & $2.27 \mathrm{e}+5$ & -- & $8.43 \mathrm{E}+4$ & $2.40 \mathrm{E}+5$ \\
\hline
\end{tabular}

\section{CONCLUSIONS}

In this paper, we proposed an effective hybrid algorithm based on genetic algorithm and ant colony optimization for continuous optimization in continuous domains. To evaluate the proposed algorithm, we have carried out a lot of simulations on several benchmark problems. Simulation results showed the proposed scheme distinctly improved the performance of $\mathrm{CGA}_{\mathrm{R}}$ and $\mathrm{ACO}_{\mathrm{R}}$, especially for the nonseparable functions and multimodal functions. The proposed algorithm has been compared with some evolutionary algorithms. From the results, we can see that the proposed algorithm outperforms the other algorithms.

\section{ACKNOWLEDGMENT}

This work was supported by Natural Science Foundation Project of CQ CSTC (No.cstc2012jjA40041) and Science Research Fund of Chongqing Technology and Business University (No. 2011-56-05).

\section{REFERENCES}

[1] K. Deep and M. Thakur, "A new crossover operator for real coded genetic algorithms," Applied Mathematics and Computation, vol.188, pp. 895-911, 2007

[2] Socha, K. \& Dorigo, M., "Ant colony optimization for continuous domains," European Journal of Operational Research, vol.185(3), pp.1155-1173, 2008.

[3] Z.Q. Chen, R.L. Wang, "A new framework with FDPP-LX crossover for real-coded genetic algorithm," IEICE Transactions on Fundamentals of Electronics, Communications and Computer Sciences, vol.E94.A(6), pp. 417-1425, 2011.

[4] Z.Q. Chen, R.L. Wang, "Two Efficient Real-coded Genetic Algorithms for Real Parameter Optimization," International Journal of Innovative Computing, Information and Control, Vol.7, No.8, pp.4871-4883, 2011.

[5] S.kobayashi, "The Frontiers of Real-Coded Genetic Algorithms," Journal of Japanese Society for Artificial Intelligence, vol.24(1), pp.128-143, 2009. In Japanese.

[6] K. Deb, A. Anand, D. Joshi, "A computationally efficient evolutionary algorithm for real-parameter evolution," Evolutionary Computation Journal, vol.10 (4), pp.371-395, 2002.

[7] Dorigo, M., \& Stützle, T, "Ant colony optimization," Cambridge, London, England: MIT Press, 2004.

[8] Dorigo, M., Maniezzo, V., \& Colorni, A, "The ant system: An autocatalytic optimizing process," Tech. Rep. 91-016 Revised, Dipartimento di Elettronica, Politecnico di Milano, Italy, 1991.

[9] Dorigo, M., Maniezzo, V., \& Colorni, A., "Ant system: Optimization by a colony of cooperating agents," IEEE Transactions on Systems, Man, and Cybernetics - Part B, Vol.26(1), pp.29-41, 1996.

[10] R. L. Wang, "A genetic algorithm for subset sum problem," Neurocomputing, vol.57, pp.463-468, 2004.

[11] J. Clerk Maxwell, A Treatise on Electricity and Magnetism, 3rd ed., vol. 2. Oxford: Clarendon, 1892, pp.68-73.
[12] R. L. WANG and Kozo OKAZAKI, "Solving Graph Planarization Problem Using an Improved Genetic Algorithm," IEICE TRANS, FUNDAMENTALS, vol.E89-A, NO.5, pp.1507-1512, 2006.

[13] R. L. WANG and Kozo OKAZAKI, "An improved genetic algorithm with conditional genetic operators and its application to set-covering problem," Soft Comput 11, pp.687-694, 2007.

[14] Dreo J, Siarry P, "A new ant colony algorithm using the heterarchical concept aimed at optimization of multiminima continuous functions," Ant Algorithms 2463:pp.216-221, 2002.

[15] Tianjun Liao, Thomas Stützle, "A unified ant colony optimization algorithm for continuous optimization," European Journal of Operational Research, vol.234, pp.597-609, 2014.

[16] Z. Michalewicz, "Genetic Algorithms + Data Structures = Evolution Programs," Springer-Verlag, New York,1992.

[17] Rosenbrock HH, "An automatic method for finding the greatest or least value of a function," The Computer Journal, vol.3(3), pp.175-184, 1960.

[18] Price KV, Storn RM, Lampinen JA, "Differential evolution: a practical approach to global optimization," Springer,2005. 